\title{
RELATION BETWEEN RADIO AND OTHER WAVELENGHTS (DISCUSSION)
}

Discussion of the paper presented by CRANE (p. 201)

Perley: The $2 \mathrm{~cm}$ VLA radio data of the western hotspot of Pictor A with comparable resolution, show an excellent correspondence between the optical and radio emission.

Crane: I would dearly love to see a comparison with HST data!

Jones D.L.: Have you looked for optical emission further out along the NGC 6251 jet, or just adjacent to the nucleus?

Crane: Unfortunately, the field of view of the FOC is quite limited. So the answer is, no I have not looked further out.

Tsvetanov: Just a brief comment on NGC 6251. Lynds and O'Neil (1994, BAAS) have shown that there is a nuclear disk in this galaxy roughly perpendicular to the radio jet axis, much like in the case of NGC 4261. I have a feeling that what you see is related to that disk, possible some edge effect.

Crane: This may be the case, but the disk you mention is considerably larger than what is seen here.

Discussion of the paper presented by WILSON (p. 205)

Bicknell: Why don't you see the other side of the helix in the NGC 4258 jets?

Wilson: Perhaps because of dust obscuration.

di Serego Alighieri: Comment: The lack of will defined ionization cones in radio-loud objects may also be due to wider opening angles, and stronger radiation pressure, which confused the picture in these objects.

Wilson: I agree that ionizing radiation may escape anisotropically from nuclei if radio galaxies, and that there are several reasons why associ- 
ated ionization cones may not be seen. If the cones have similar linear extents to those in Seyferts, they would be hard to resolve spatially at the much longer distances of FRII radio galaxies. Also, it may be that the interstellar gas in ellipticals is mostly hot and not susceptible to photoionization by the nucleus.

di Serego Alighieri: The evidence for non-ionized gas outside the ionization cones would nicely support the fact that these cones are ionization bounded. Is there any such evidence?

Wilson: Prieto and Freudling (1993) have mapped the $21 \mathrm{~cm} \mathrm{HI}$ emission of NGC 5252. They find that the neutral hydrogen tends to avoid the ionization cones and that there is evidence for a continuity of velocity from the ionized gas within the cones to the neutral gas outside them. 'i'heir results support the idea that pre-existing neutral gas is ionized by an anisotropic radiation field, i.e. the cones are ionization-bounded.

Macchetto: In the sample of Seyfert 2 galaxies we have observed with HST (see poster by Capetti et al), we find that for the Seyfert 2s with weak jets i.e. where there are lobes well within the galaxy, we appear to need an additional ionizing source to explain the increase in ionization potential with distance from the nucleus and the high-ionization ([OIII $] /[\mathrm{OII}])$ seen in the bright filaments. We believe that in these cases, shocks are the best explanation as they are expected, they reproduce the observed morphology and are the simplest explanation.

Wilson: I think the issue of whether the gas in the NLR and ENLR of Seyfert 2 galaxies is photoionized by a compact nuclear source or by radiation from high velocity shocks in the NLR is still unresolved. The main process may differ in different galaxies - e.g. Seyfert 2's with hidden Seyfert 1's should have strong, compact nuclear ionizing sources, while in Seyfert 2's without Seyfert 1 nuclei (if such objects exist), photoionizing shocks in the NLR may dominate. Of course, the compact nuclear ionizing source may itself result from high velocity shocks.

Discussion of the paper presented by MORAN (p. 211)

Yu Zhi-Yao: Could you find the effect in $\mathrm{OH}$ megamaser?

Moran: The effect has not been found in $\mathrm{OH}$ megamaser because of the different resolution.

Wilson: As you have shown, it is likely that the maser features near systemic velocity result from amplification of radio continuum photons from 
a central radio source. What do you think is the source of photons for the high velocity "satellite lines"?

Moran: Of course, it's just a hypothesis that the systemic features amplify a central continuum source, since we have not been able to detect this source directly. We assume that the high velocity features are unsaturated and amplify their own spontaneous emission.

Gelderman: What is the physical reason why the masers are seen only either along the line of sight or at a right angle to the line of sight.

Moran: We are most likely to see masers along directions through the disk where the coherent amplification path length is greatest. This path length is the distance over which the velocity changes by less than the thermal linewidth. For a keplerian velocity field, in a disk viewed edge on, the velocity gradient is zero, and hence the path length maximum, along the direction to the center of the disk and along the disk diameter that is perpendicular to the line of sight.

Discussion of the paper presented by TADHUNTER (p. 217)

Wall: These objects are from flux-limited samples; hence by 'low redshift' objects you mean objects of low radio luminosity, and by high redshift you mean high-luminosity, do you not?

Tadhunter: Yes, the high redshift objects selected from flux-limited samples inevitably have higher radio luminosities and jet powers. I believe that many of the observed differences between high and low redshift objects could be a consequence of this fact, although there remains the possibility that the evolution in the ISM is also important.

Leahy: It seems that one can identify jet-cloud interactions quite easily by their dramatic effect on the radio structure - certainly in $3 \mathrm{C} 171$. High redshift objects like 3C294 may be analogous.

Tadhunter: The radio structures observed in $3 \mathrm{C} 171$ are certainly unusual in their degree of distortion, but there are also objects like PKS 2250-41 which have relatively normal double structures despite showing compelling evidence for jet/cloud interactions.

Laing: If you attribute most of the aligned continuum in high-z galaxies to jet/shock effects (as in your hybrid model), do you still get the observed polarization, or is there too much dilution of the polarized component? 
Tadhunter: In fact, the dilution by the nebular continuum helps to explain why the observed polarization is less than predicted for pure dust scattered light. The uncertainties in the estimated nebular continuum and off-nuclear polarization are such that it is too early to say whether there is too much dilution.

Wilson: Could you tell us the ratios of $[0 I I I] \lambda 4363 /[0 I I I] \lambda 5007$ (or alternatively the electron temperatures) that you observe? I think its useful to bear in mind that $4363 / 5007$ ratios at least as high as 0.02 are expected in the matter-bounded plus ionization-bounded photoionization models that Luc Binette described this morning.

Tadhunter: The $[0 \mathrm{III}] 4363 /(5007+4959)$ ratio falls in the range $0.01-$ 0.03 for the EELR, corresponding to electron temperatures in the range $13,000-22,000 \mathrm{k}$ (low density limit). There is already good evidence that matter-bounded components are important in some aspects (eg. Tadhunter et al. 1988, MNRAS, 235, 403, PKS 2152-69), but for low ionization EELR there would be a problem with explaining the relatively weak $\mathrm{HeII}(4686)$ and the relatively strong $[0 \mathrm{I}](6300)$ with a model which included a significant matter-bounded component.

Discussion of the paper presented by MORGANTI (p. 227)

Bicknell: The difference in velocity widths of the high excitation over low excitation lines has a natural exploration in terms of shock models. [OIII] for example would arise from the shock precursor whereas the [OI] would come from the more turbulent region behind the shock. The radiative cooling zone would be too small for it to be resolvable.

Morganti: I agree that the kinematic differentiation is difficult to explain in terms of control source photoionization but could be entirely consistent with ionization by fast shocks.

Urry: Your [OIII] image looks very similar to the ionization cones in radioquiet AGN shown earlier this morning by Wilson and Tsvetanov, for example, and the axis is well aligned with the radio structure as you pointed out - is this the first example of an ionization cone (two-sided, no less!) in a radio-loud object? How does it compare to the ionization cones in radio-quiet AGN?

Morganti: Actually, I have not really looked at it as an ionization cone. I think that the nice coincidence between the [OIII] emission and the radio continuum (not very common in radio galaxies) looks more like 
the result of an interaction, therefore supporting the important role of jet plasma, more than photoionization, in ionizing the gas.

Discussion of the paper presented by BINETTE (p. 232)

Simkin: How much neutral material will you predict. We find (Simkin \& Collaef -poster) that $\mathbf{3 4 9 - 2 7}$ has very little HII. Is this consistent with your model?

Binette: I estimate the column density of neutral $\mathrm{HI}$ from the ionization bounded component to be less or equal to $3 \times 10^{20} \mathrm{~cm}^{-2}$. I would need the area of the emitting clouds to convert this into a mass of $\mathrm{HI}$.

Macchetto: In your model the assumption that the "ionization bound" clouds are always behind "matter bound" clouds appears to be contrived. In a natural, real life situation, I would expect a mixing of the two types at all spatial distances. What would be the spectral characteristics in this case?

Binette: We are mixing the two types at all distances. However, what we claim is that the low excitation clouds are such because they see a modified ionizing continuum and not the result of arbitrarily reducing the ionization parameter. This is also the only way to get the low $\mathrm{HeII} / \mathrm{H} \beta$ observed in some objects. in other words, we propose that whenever a cloud sees a pure power law, this leads to a matter bounded component with high excitation spectrum. This whatever the distance from the source.

Discussion of the paper presented by LEDLOW (p. 238)

Gavazzi: Is there any evidence for a correlation between radio loudness and the presence of some nuclear enhancement in the optical?

Ledlow: No. Comparisons of luminosities measured for small radii and larger apertures show no enhancement in the nuclear luminosities for radio-loud vs. radio-quiet ellipticals.

Laing: What do you think is the source of the discrepancy between your results and those of Disney et al., who claimed that radio galaxies are rounder and redder than "normal" ellipticals, and live in richer environments? Are there real differences (eg in cluster/non-cluster location) or is it that you have been more careful with your control sample? 
Ledlow: The analysis from CCD data may be more accurate, but I suspect that there may be some intrinsic differences in the galaxies in and out of rich clusters. This has been suggested by de Carvalho and Djorgovski (1992) and others. However, if true, these differences don't appear to affect the observed radio luminosity function. Upon reflection, I don't think there is a problem with Disney et al.'s control sample.

Menon: Your results are very similar to my results (Menon, 1992 M.N) concerning radio sources in compact groups of galaxies except for one difference. I found a strong correlation between nearest neighbour distance and the occurrence of radio emission in the brightest galaxy in the group. This is understandable since the crossing times in groups are one or two orders of magnitude smaller and velocity dispersions are about $200 \mathrm{Km} / \mathrm{sec}$.

Ledlow: I agree that the differences are most likely related to the differences in velocity dispersion and the dynamics of poor group systems.

Discussion of the paper presented by IMPEY (p. 281)

Pohl: Egret sources identified with flat-spectrum radio quasars are a strongly flux-limited sample, both in $\gamma$-rays and in the radio regime. The available flux range in both regimes is around one decade, similar to the variability range in the $\gamma$-ray range. Comparing simultaneous and simultaneous data there is no direct relation in radio and $\gamma$-ray flux; therefore any estimate of the $\gamma$-ray background as superposition of AGN is highly uncertain.

Impey: The analysis assumes a factor of 3-4 rms variability in both radio and $\gamma$-ray flux, so this uncertainty is incorporated into the analysis. There is formally a correlation for the EGRET $1 \mathrm{Jy}$ sources at the $98 \%$ confidence level; the deduced contribution to the $\gamma$-ray background of course has a large error bar.

Perlman: To follow up on the previous question: There have now been two detections by EGRET of $<1$ Jy radio sources MKN421 and 2155304. There are also a large number $(>40)$ unidentified EGRET sources. These are likely all $<1 \mathrm{Jy}$ sources as well.

Impey: That's basically right 
Discussion of the paper presented by M.F. ALLER (p. 283)

Ekers: Is the correlation with polarization variability better than with I?

Aller: In a few cases individual events are better resolved in polarized flux than in total flux, but the difficulty in establishing a meaningful correlation is due to the insufficient sampling in the $\gamma$-ray region.

Ekers: Is it possible that the polarization variability during "quiescent" times is due to variations in the depolarizing plasma which may be affected by the $\gamma$-ray activity?

Aller: No. We have not seen any evidence for time-variable depolarization.

Discussion of the paper presented by NORMAN (p. 291)

Begelman: Granted that the EeV baryons from cosmological distances cannot reach us, wouldn't the distant sources give us a high-energy neutrino background that we might be able to detect?

Norman: Yes, the background limits due to neutrinos from such processes may give interesting limits.

Vietri: The proton mean free path against photo-pion destruction above $10^{20} \mathrm{eV}$ is less than $20 \mathrm{kpc}$ combined with the near isotropy of the UHE$\mathrm{CRs}$ detection of arrival, and their slight concentration toward the Supergalactic plane, indicate that only galaxies can produce UHECRs. Normal galaxies do not produce UHECRs, but they contain GRBs, so I see this as (slight) evidence for GRBs.

Norman: Yes, this is an interesting line of argument in favor of the GRB / UHECR hypothesis.

Gopal-Krishna: Since propagation losses on high energy cosmic rays are so severe, their detection would be biased heavily in favor of the one that travelled straight toward us. Perhaps we need not be so pessimistic about spatial correlation between the detached events and their sources?

Norman: Yes, this is reasonable. The Auger array will provide sufficiently high quality data to confirm this or otherwise.

Rudnick: There is considerable uncertainty in the physical parameters of extragalactic sources. Which are the critical parameters for us to pin down to evaluate their role in high energy cosmic ray acceleration? 
Norman: The crucial parameter is the magnetic field strength.

Leahy: Shock acceleration requires a suprathermal component of the initial particle distribution which may be hard to arrange for the unprocessed material you need, except for the case of shocks within radio sources, where the AGN has provided the seed population. After all, these are the only systems you discussed which show synchrotron radiation.

Norman: I assume in our calculation that the injection problem solves itself.

Ekers: You need the mean free path to limit the volume of space for the sources.

Norman: There could be a hard spectrum source beyond the limit as discussed by Sigl et al. 1995.

Woltjer: Even if one believes the composition change at $10^{10} \mathrm{eV}$, isn't it true that one knows nothing about the composition of the $10^{21} \mathrm{eV}$ events? Moreover, since any cosmic ray acceleration scheme needs injection processes which may be composition dependent can we really be confident that the source region must be metal deficient, even if the CR were?

Norman: First, I agree the metallicity-composition argument is not watertight but it seems interesting and subject to increasingly detailed experimental test with, for example, the Auger array. Second, I also agree the very highest energy events have at present no constraints at all on their composition. 